\title{
Focal dose escalation using FDG-PET-guided intensity-modulated radiation therapy boost for postoperative local recurrent rectal cancer: a planning study with comparison of DVH and NTCP
}

Keiichi Jingu ${ }^{1 *}$, Hisanori Ariga' ${ }^{1}$, Tomohiro Kaneta², Yoshihiro Takai ${ }^{1}$, Ken Takeda ${ }^{1}$, Lindel Katja ${ }^{4}$, Kakutaro Narazaki ${ }^{1}$, Takahiro Metoki ${ }^{1}$, Keisuke Fujimoto', Rei Umezawa', Yoshihiro Ogawa', Kenji Nemoto³, Masashi Koto', Masatoshi Mitsuya', Naruhiro Matsufuji ${ }^{5}$, Shoki Takahashi ${ }^{2}$, Shogo Yamada ${ }^{1}$

\begin{abstract}
Background: To evaluate the safety of focal dose escalation to regions with standardized uptake value (SUV) $>2.0$ using intensity-modulated radiation therapy (IMRT) by comparison of radiotherapy plans using dose-volume histograms (DVHs) and normal tissue complication probability (NTCP) for postoperative local recurrent rectal cancer

Methods: First, we performed conventional radiotherapy with $40 \mathrm{~Gy} / 20$ fr. (CRT $40 \mathrm{~Gy}$ ) for 12 patients with postoperative local recurrent rectal cancer, and then we performed FDG-PET/CT radiotherapy planning for those patients. We defined the regions with SUV > 2.0 as biological target volume (BTV) and made three boost plans for each patient: 1) CRT boost plan, 2) IMRT without dose-painting boost plan, and 3) IMRT with dose-painting boost plan. The total boost dose was 20 Gy. In IMRT with dose-painting boost plan, we increased the dose for BTV+5 $\mathrm{mm}$ by $30 \%$ of the prescribed dose. We added CRT boost plan to CRT 40 Gy (summed plan 1), IMRT without dosepainting boost plan to CRT 40 Gy (summed plan 2) and IMRT with dose-painting boost plan to CRT 40 Gy (summed plan 3), and we compared those plans using DVHs and NTCP.

Results: $D_{\text {mean }}$ of PTV-PET and that of PTV-CT were 26.5 Gy and 21.3 Gy, respectively. $V_{50}$ of small bowel PRV in summed plan 1 was significantly higher than those in other plans ((summed plan 1 vs. summed plan 2 vs. summed plan 3: $47.11 \pm 45.33 \mathrm{~cm}^{3}$ vs. $40.63 \pm 39.13 \mathrm{~cm}^{3}$ vs. $41.25 \pm 39.96 \mathrm{~cm}^{3}(p<0.01$, respectively)). There were no significant differences in $V_{30}, V_{40}, V_{60}, D_{\text {mean }}$ or NTCP of small bowel PRV.
\end{abstract}

Conclusions: FDG-PET-guided IMRT can facilitate focal dose-escalation to regions with SUV above 2.0 for postoperative local recurrent rectal cancer.

\section{Background}

Although positron emission tomography using ${ }^{18} \mathrm{~F}$-fluorodeoxyglucose (FDG-PET) has become widely used for diagnosis of various malignant tumors, the spatial resolution of PET images alone is not high and it is difficult to determine anatomical sites in detail. However, this problem has been solved by the use of a combined

\footnotetext{
* Correspondence: kjingu-jr@rad.med.tohoku.ac.jp
'Department of Radiation Oncology, Tohoku University School of Medicine,

*Correspondence: kjingu-jr@rad.med.tohoku.ac.jp
'Department of Radiation Oncology, Tohoku University School of Medicine, Sendai, Japan
}

(c) 2010 Jingu et al; licensee BioMed Central Ltd. This is an Open Access article distributed under the terms of the Creative Commons Attribution License (http://creativecommons.org/licenses/by/2.0), which permits unrestricted use, distribution, and reproduction in any medium, provided the original work is properly cited. images to be obtained at almost the same time and at the same position.

Local recurrence rates of rectal cancer after surgery including dissection of lateral nodes have been reported to be about $9 \sim 12 \%$ in Japan [1-3], and the prognosis after local recurrence is poor. In the case of local recurrence, the best salvage treatment for achieving long-term local control and survival is total pelvic exenteration with distal sacrectomy. The 5-year overall 
survival rate in patients after $\mathrm{R} 0$ resection has been reported to be $30 \sim 40 \%[4,5]$. Since about half of the patients with local recurrent rectal cancer die due to only local lesions without distant metastasis [6], local control would be beneficial for survival. However, extended surgery is not widely used because of high morbidity and mortality rates. Moreover, it has been pointed out that total pelvic exenteration reduces the quality of life of patients. Furthermore, Tepper et al. reported that only $34 \%$ of patients with locally or distantly recurrent rectal cancer could receive a potentially curative resection [7]. In Japan, due to the lower rate of local recurrence after surgery alone, induction radiotherapy is not performed in most patients [8]. And, based on SEER, over $30 \%$ of patients with advanced-stage rectal cancer in the United States also did not undergo radiation therapy [9]. Therefore, external body radiotherapy is one of the most widely used therapies and provides good palliation of pain in $50 \sim 80 \%$ of patients with postoperative local recurrence; however, it has a poor survival benefit [10]. We have been performing conventional irradiation for postoperative local recurrent lesions with a total dose of $60 \mathrm{~Gy}(2 \mathrm{~Gy} /$ fraction $\cdot 5$ fractions/week), but we have considered that dose escalation is necessary to cure patients because rectal cancer has many hypoxic fractions [11]. In fact, some studies have revealed that local failure rate after radiotherapy alone decreased with increasing irradiation dose $[12,13]$. However, dose escalation with conventional radiotherapy is difficult due to the location of critical organs (e.g, small bowel) around the lesion.

Huebner et al. showed by a meta-analysis that the sensitivity, specificity and accuracy of FDG-PET for local recurrent rectal cancer were $94.5 \%, 97.7 \%$ and $95.9 \%$, respectively [14]. FDG-PET is superior to conventional modalities (e.g, CT and MRI) for distinguishing between local recurrence and postoperative scar. There have been several reports recently on the usefulness of FDGPET for radiotherapy planning in lung cancer and head and neck cancer. FDG-PET has been reported to be useful for delineation of target gross tumor volume (GTV) or clinical target volume (CTV).

We performed FDG-PET/CT planning in 12 patients with postoperative local recurrent rectal cancer during conventional radiotherapy at $40 \mathrm{~Gy}$.

As a preclinical study, we planned focal dose escalation to high FDG uptake regions in those 12 patients with intensity-modulated radiation therapy (IMRT) in the radiotherapy planning system, and we compared the IMRT plans with conventional radiotherapy plans in dose-volume histograms (DVHs) and normal tissue complication probability (NTCP).

The purpose of the present study is to evaluate the safety of focal dose escalation to regions with standardized uptake value (SUV) above 2.0 using IMRT in DVH and NTCP in patients with postoperative locoregional recurrent rectal cancer

\section{Methods \\ Criteria for eligibility}

Eligibility criteria were as follows: (1) postoperative locoregional recurrent rectal cancer, (2) unresectable, (3) age between 20 and 79 years, (4) Karnofsky Performance Status (KPS) score of $>=60$, (4) without distant metastasis, (5) tumor is grossly measurable, and (6) no serious medical or psychologic conditions precluding safe administration of treatment.

\section{Radiotherapy}

A linear accelerator (Clinac 23EX (VARIAN Medical Systems, Palo Alto, CA), 6 or 15 MV) was used as the $\mathrm{X}$-ray source.

First, we performed radiotherapy planning using CT with contrast medium for 12 patients with postoperative locoregional recurrent rectal cancer. All target volumes were outlined slice by slice on the treatment-planning CT images. GTV was defined as the gross extent of the tumor shown by imaging as well as physical examination, CTV was defined as GTV plus a $10-\mathrm{mm}$ circular margin for potential microscopic spread, and planning target volume (PTV) was defined as CTV plus a $5-\mathrm{mm}$ circular margin to account for organ motion and patient setup errors. Additionally, we attached a 5-mm leaf margin to PTV. The patients were prescribed $40 \mathrm{~Gy}$ in 20 fractions with the dose prescribed to the isocenter (CRT 40 Gy) using a median of 4 (range 3-4) coplanar irradiation fields.

Next, we performed FDG-PET/CT with a carbon graphite flat tabletop in the supine position for radiotherapy planning at $40 \mathrm{~Gy}$ in the same 12 patients. The images obtained by CT and PET were sent to the radiation therapy planning system as DICOM data, and the CT and PET images were fused using DICOM information. Residual gross extent of the tumor shown in CT images at 40 Gy was defined as GTV2, CTV-CT was defined as GTV2 plus a 5-mm circular margin, and PTV-CT was defined as CTV-CT plus a 5-mm circular margin. We defined the regions with standardized uptake value (SUV) above 2.0 as biological target volume (BTV) and BTV $+5-\mathrm{mm}$ circular margin as PTV-PET. Our radiotherapy planning system could show the degree of FDG accumulation with not SUV but $\mathrm{Bq} / \mathrm{ml}$ in PET images. If the total dose of FDG administered to the patient and the patient's body weight are known, we can define regions with an arbitrary range of SUVs even in our radiotherapy planning system by adjusting the window level and range. In the present study, we delineated BTV under the condition showing SUV of 2.0 to 20.0 . 


\section{Protocol of the present planning study}

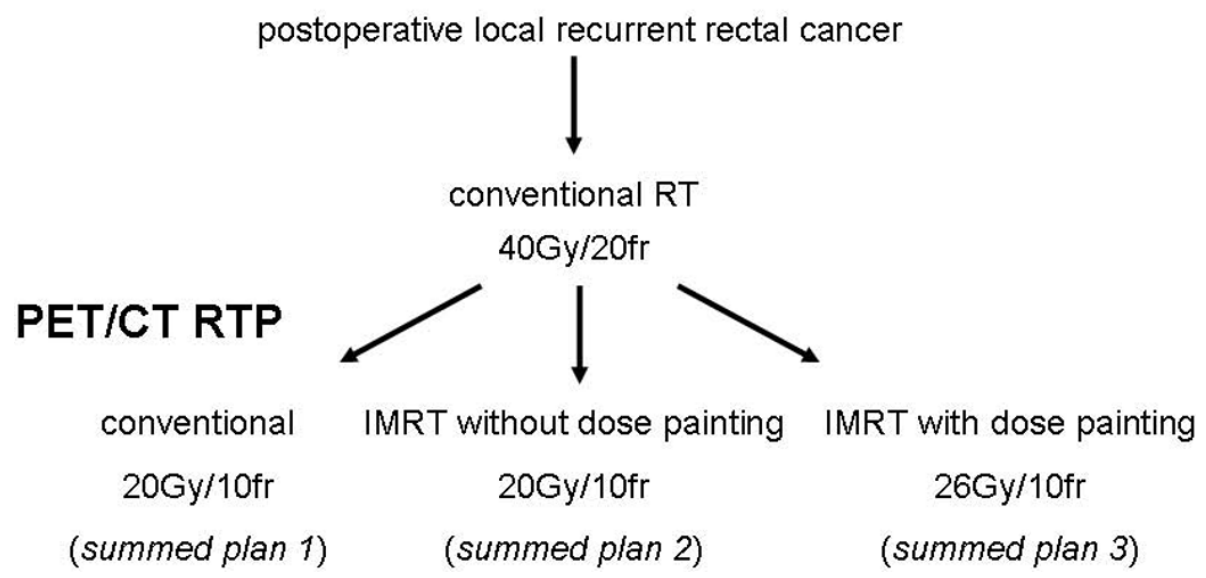

Figure 1 Pattern diagram of the present planning study.

We made three boost plans for each patient: 1) conventional radiotherapy plan (CRT boost plan), 2) IMRT plan not using dose painting (IMRT without dose-painting boost plan), and 3) IMRT plan using dose painting (IMRT with dose-painting boost plan) (Figure 1). The fractional dose of radiotherapy was 2.0 Gy with normalization at $95 \%$ of PTV-CT, and the total boost dose was 20.0 Gy. In IMRT dose-painting boost plan, we increased the dose of PTV-PET by $30 \%$ of the prescribed dose ( 2.6 Gy/fraction, total 26.0 Gy) using dose-painting.

We defined the small bowel as the organ at risk (OAR) because the small bowel is the most vulnerable to radiation in pelvic organs, and we carefully delineated the whole small bowel in the abdomen and pelvis, preferably with the colon, bladder or other organs with reference to CT using contrast medium. The planning organ at risk volume (PRV) was margined with $5 \mathrm{~mm}$ to the OAR as with the PTV margin.

IMRT plans were generated using Varian Eclipse (Helios IMRT) Workstations. The beam arrangement consisted of seven coplanar non-colinear fields $\left(30^{\circ}, 80^{\circ}\right.$, $130^{\circ}, 180^{\circ}, 230^{\circ}, 280^{\circ}, 330^{\circ}$ ), and delivery of IMRT was carried out using the sliding window technique with a 15 MV linear accelerator equipped with a dynamic multileaf collimator. Our primary inverse planning objectives were as follows: (1) uniform dose of 20 Gy to PTV-CT (relative weight, $\mathrm{w}=15)$, (2) maximal irradiated dose (Dmax) of small bowel PRV $<20$ Gy ( $w=$ 20) (total Dmax of small bowel PRV $<60$ Gy), (3) dose received by $5 \%$ (D5) of small bowel PRV $<15$ Gy (w $=8$ ) (total D5 of small bowel PRV $<55$ Gy), and in the IMRT with dose-painting boost plan, we added (4) dose received by $95 \%$ (D95) of PTV-PET $>26$ Gy $(\mathrm{w}=10)$ (total D95 of PTV-PET >66 Gy, which means normalized 2-Gy-equivalent biologically effective dose, 67.3 Gy, calculated using an alpha/beta value of 10).

IMRT planning for this study was theoretical and was not used for treatment. All of the patients underwent conformal radiation therapy to a total dose of $60 \sim 66 \mathrm{~Gy}$ (2.0 Gy/fraction/day).

We added each boost plan to CRT 40 Gy (summed plan 1: CRT $40 \mathrm{~Gy}+$ CRT boost plan, summed plan 2: CRT 40 Gy + IMRT without dose-painting boost plan, summed plan 3: CRT 40 Gy + IMRT with dose-painting boost plan) (Figure 2) and we compared those plans using DVHs and NTCP of small bowel PRV.

The differences in dose distribution between IMRT without dose-painting boost plan and IMRT with dosepainting boost plan were evaluated by the conformity index (C.I.), which we defined as the ratio of the volume irradiated with $95 \%$ of the prescribed dose to PTV-CT.

\section{NTCP}

NTCP was calculated using the Lyman-Kutcher-Burman model.

$$
\begin{aligned}
& \mathrm{NTCP}=1 / \sqrt{2 \pi} \int_{-\infty}^{t} \exp \left(-\chi^{2} / 2\right) d x \\
& \mathrm{t}=\left(\mathrm{D}-\mathrm{TD}_{50}(v)\right) /\left(\mathrm{m} \mathrm{TD}_{50}(v)\right) .
\end{aligned}
$$

Irradiation of fractional volume $v=\mathrm{V} / \mathrm{V}_{\text {ref }}$ corresponds to parameter TD50 (v) for 50\% complication probability, given by the following power law:

$$
\mathrm{TD}_{50}(v)=v^{-\mathrm{n}} \mathrm{TD}_{50}
$$




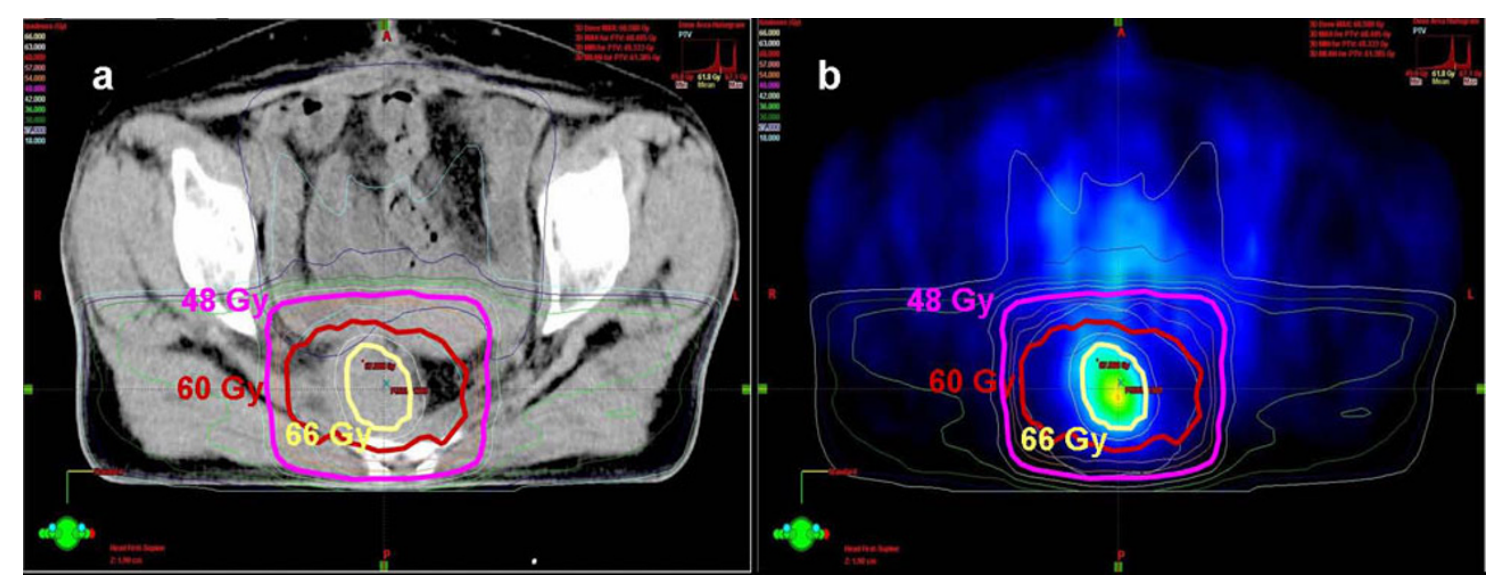

Figure 2 Dose distribution of summed plan 3 (summed plan of CRT 40Gy and IMRT with dose-painting boost plan) in a patient. a): dose distribution in $C T$, b): dose distribution in FDG-PET.

$\mathrm{V}_{\text {ref }}$ is the total volume of the organ.

With inhomogeneous irradiation, there are multiple partial volume irradiations to different doses. The effects of partial volume irradiations are computed according to the Kutcher-Burman (K-B) effective-volume dosevolume histogram reduction scheme [15]. The K-B method satisfies the consistency requirement that partitioning a uniform irradiation of the entire volume into multiple partial volume irradiations to the same dose results in the same NTCP. The organ dose is described as independent fractional volume elements $v_{j},(j=1, \ldots$

$k), \sum_{j=1}^{k} v_{j}=1$, irradiated to doses $\mathrm{d}_{\mathrm{j}}$. Then, with the K-B algorithm, an effective fractional volume $\mathrm{V}_{\text {eff(j) }}$ defined as follows is computed for each $v_{j}$. The effective fractional volume is the volume that, when irradiated to reference dose $d_{\text {ref }}$, would give the same complication probability in the Lyman model as the actual fractional volume $v_{\mathrm{j}}$ irradiated to dose $\mathrm{d}_{\mathrm{j}}$. If $v_{\mathrm{j}}$ were the only fractional volume irradiated, then from equality of effect and Eq. (B), it would follow that

$$
\mathrm{V}_{\mathrm{eff}(\mathrm{j})}=v_{j}\left(\mathrm{~d}_{\mathrm{j}} / \mathrm{d}_{r e f}\right)^{1 / \mathrm{n}} \text {. }
$$

In the K-B method, Eq. (D) is applied to all fractional subvolumes. In the present study, $\mathrm{d}_{\text {ref }}$ was chosen to be the maximum dose $D_{\max }$. The total effective fractional volume receiving the reference dose is calculated as follows:

$$
V_{\text {eff }}=\sum_{j=1}^{k} V_{e f f(j)}
$$

using $v=\mathrm{V}_{\text {eff }}$ in Eq. (C) and $\mathrm{D}=\mathrm{d}_{\text {ref }}=\mathrm{D}_{\max }$ in Eq. (B)

The parameters used to calculate small bowel obstruction and perforation were $n=0.15, m=0.16$, and $\mathrm{TD}_{50}$ $=55[16,17]$.

\section{Chemotherapy}

For all of the 12 patients, S-1 $60 \mathrm{mg} / \mathrm{m}^{2}$ was given orally twice daily (within 30 minutes after morning and evening meals) for 2 weeks, followed by a drug-free interval of one week (one cycle) concomitant with radiation therapy. Chemotherapy was not performed for a period of at least 4 weeks before initiation of radiation therapy in any of the patients.

\section{FDG-PET}

PET scans were performed 1 hour after administration of ${ }^{18} \mathrm{~F}$-fluorodeoxyglucose at a dose of $3.1 \mathrm{MBq} / \mathrm{kg}$ using a Biograph PET/CT scanner (Siemens, Hoffman Estates, IL) under the condition of more than 4 hours of fasting. A transmission scan was performed for attenuation correction before the emission scans (using a computed tomography scan). Seven bed positions were used for emission scans, with an acquisition time of 2 minutes per position. For radiotherapy planning, PET/CT scans were performed in the same posture as that for treatment on a flat carbon-fiber table top. The PET images were reconstructed with an ordered-subset expectation maximization (OSEM) iterative reconstruction algorithm.

For semiquantitative analysis of increased FDG uptake lesions, SUV based on body weight (g) was calculated and converted into a value based on lean body mass:

SUV $=[$ tissue activity concentration $(\mathrm{Bq} / \mathrm{ml})] /[$ administered activity $(\mathrm{Bq}) /$ weight $(\mathrm{g})]$.

The blood glucose levels of all patients before scans were less than $150 \mathrm{mg} / \mathrm{dl}$.

\section{Statistical analysis}

Statistical significance was defined as a value of $\mathrm{p}<0.05$ in the present study. SPSS software for Windows version 11.0 (SPSS Inc, Chicago, IL) was used for all calculations. Multiple pairwise comparisons were performed by 
using one-way analysis of variance $t$-test with the Bonferroni method.

\section{Ethics}

The present study protocol was reviewed and approved by the Ethics Committee of Tohoku University Graduate School of Medicine (approval number, 2007-418), and informed consent was obtained from all patients before radiation therapy.

\section{Results}

The results of comparison of the plans in all 12 patients are shown in Additional file 1; Table S1. Even with the fusion method using DICOM information, there were no significant displacements between PET images and CT images. We did not need to fuse them manually again. The locations of the highest level of FDG accumulation after $40 \mathrm{~Gy}$ in local recurrent regions were almost the same as those before radiation therapy in the 12 patients, but maximal SUV decreased significantly from $6.84 \pm 3.25$ before radiation therapy to $5.14 \pm 2.81$ at $40 \mathrm{~Gy}(\mathrm{p}=0.035$, Wilcoxon's test). Figure 3 shows change in FDG accumulation caused by irradiation of 40 Gy in a patient with anastomotic recurrence. In the present study, although there was no significant difference between GTV and GTV2 (GTV vs. GTV2: $87.52 \pm$ $63.06 \mathrm{~cm}^{3}$ vs. $\left.79.66 \pm 57.80 \mathrm{~cm}^{3}, \mathrm{p}=0.141\right)$, there was a significant difference between GTV2 and BTV (GTV2 vs. BTV: $79.66 \pm 57.80 \mathrm{~cm}^{3}$ vs. $11.12 \pm 21.92 \mathrm{~cm}^{3}, \mathrm{p}<$ 0.001) (Additional file 1; Table S2). In the IMRT with dose-painting boost plan, mean irradiated dose $\left(D_{\text {mean }}\right)$ of PTV-PET and that of PTV-CT were $26.5 \pm 0.8$ Gy and $21.3 \pm 0.8 \mathrm{~Gy}$, respectively.

With regard to the volume of small bowel PRV receiving 50 Gy or more $\left(\mathrm{V}_{50}\right)$, there were significant differences between summed plan 1 and summed plan 2 and between summed plan 1 and summed plan 3 (summed plan 1 vs. summed plan 2 vs. summed plan 3: $47.11 \pm$ $45.33 \mathrm{~cm}^{3}$ vs. $40.63 \pm 39.13 \mathrm{~cm}^{3}$ vs. $41.25 \pm 39.96 \mathrm{~cm}^{3}$ $(\mathrm{p}<0.01$, respectively)) (Additional file 1 ; Table S2).

With regard to the volume of small bowel PRV receiving 60 Gy or more $\left(\mathrm{V}_{60}\right), 40$ Gy or more $\left(\mathrm{V}_{40}\right), 30$ Gy or more $\left(\mathrm{V}_{30}\right)$ and $\mathrm{D}_{\text {mean }}$ of small bowel PRV, there were no significant differences (summed plan 1 vs. summed plan 2 vs. summed plan $3: \mathrm{V}_{60}, 19.76 \pm 23.67 \mathrm{~cm}^{3}$ vs. $13.65 \pm 18.88 \mathrm{~cm}^{3}$ vs. $14.52 \pm 19.18 \mathrm{~cm}^{3} ; \mathrm{V}_{40}, 77.32 \pm$ $64.21 \mathrm{~cm}^{3}$ vs. $71.33 \pm 60.20 \mathrm{~cm}^{3}$ vs. $72.55 \pm 61.59 \mathrm{~cm}^{3}$; $\mathrm{V}_{30}, 121.18 \pm 119.6 \mathrm{~cm}^{3}$ vs. $113.62 \pm 99.69 \mathrm{~cm}^{3}$ vs. $116.88 \pm 104.94 \mathrm{~cm}^{3} ; \mathrm{D}_{\text {mean }}, 16.1 \pm 5.8$ Gy vs. $16.4 \pm 5.6$ Gy vs. $16.6 \pm 5.8$ Gy (n.s.)) (Figure 4 and 5, Additional file 1; Table S2).

Focal dose escalation using dose-painting slightly but significantly increased maximal irradiated dose $\left(D_{\max }\right)$ of small bowel PRV(summed plan 1 vs. summed plan 2 vs. summed plan 3: $55.0 \pm 16.0$ Gy vs. $54.9 \pm 15.3$ Gy vs. $57.4 \pm 16.3$ Gy $(\mathrm{p}<0.01$, respectively)); however NTCP of small bowel PRV was not significantly increased even using focal dose escalation (summed plan 1 vs. summed plan 2 vs. summed plan 3: $5.10 \pm 5.66 \%$ vs. $3.78 \pm$ $4.19 \%$ vs. $4.09 \pm 4.62 \%$ ) (Additional file 1 ; Table S2). In the 4 patients with lateral pelvic lymph node metastasis or perineum recurrence, there were no significant

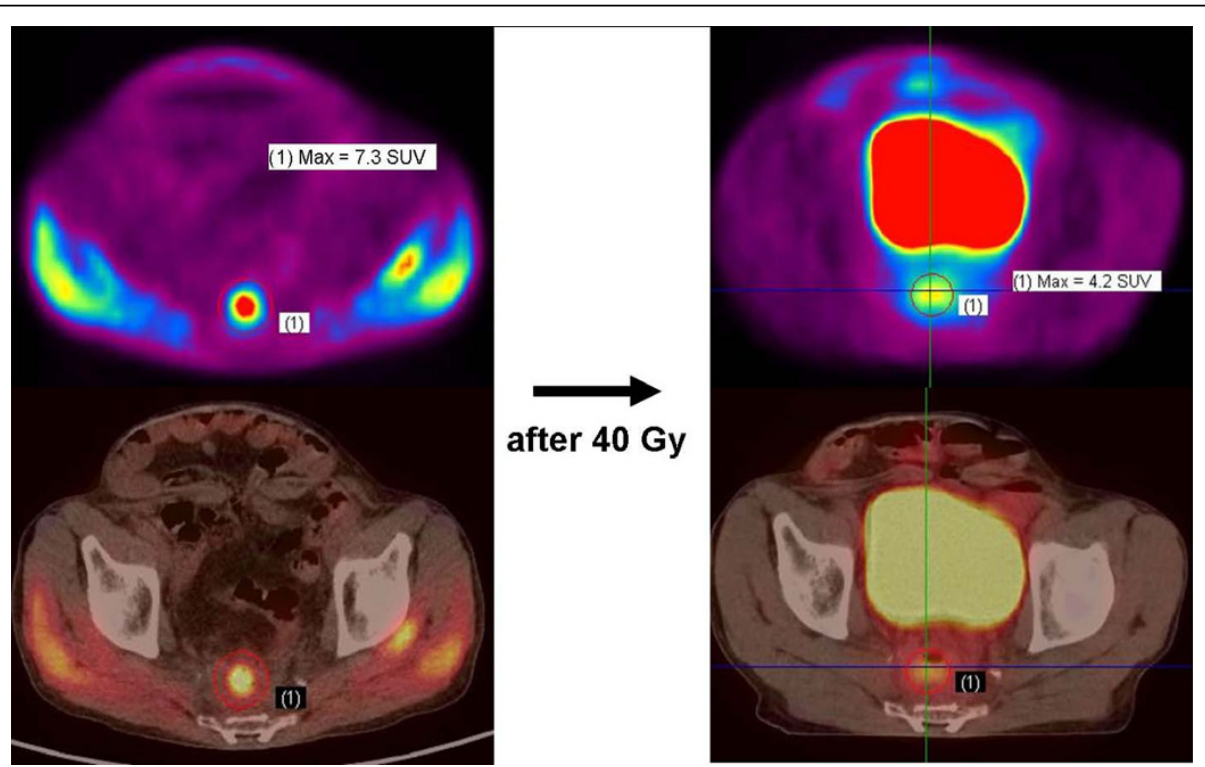

Figure 3 Change in FDG accumulation. This patient had anastomatic recurrence with 7.3 SUVmax before radiation therapy. After 40 Gy, the accumulation was decreased by 3.1 SUVmax. 


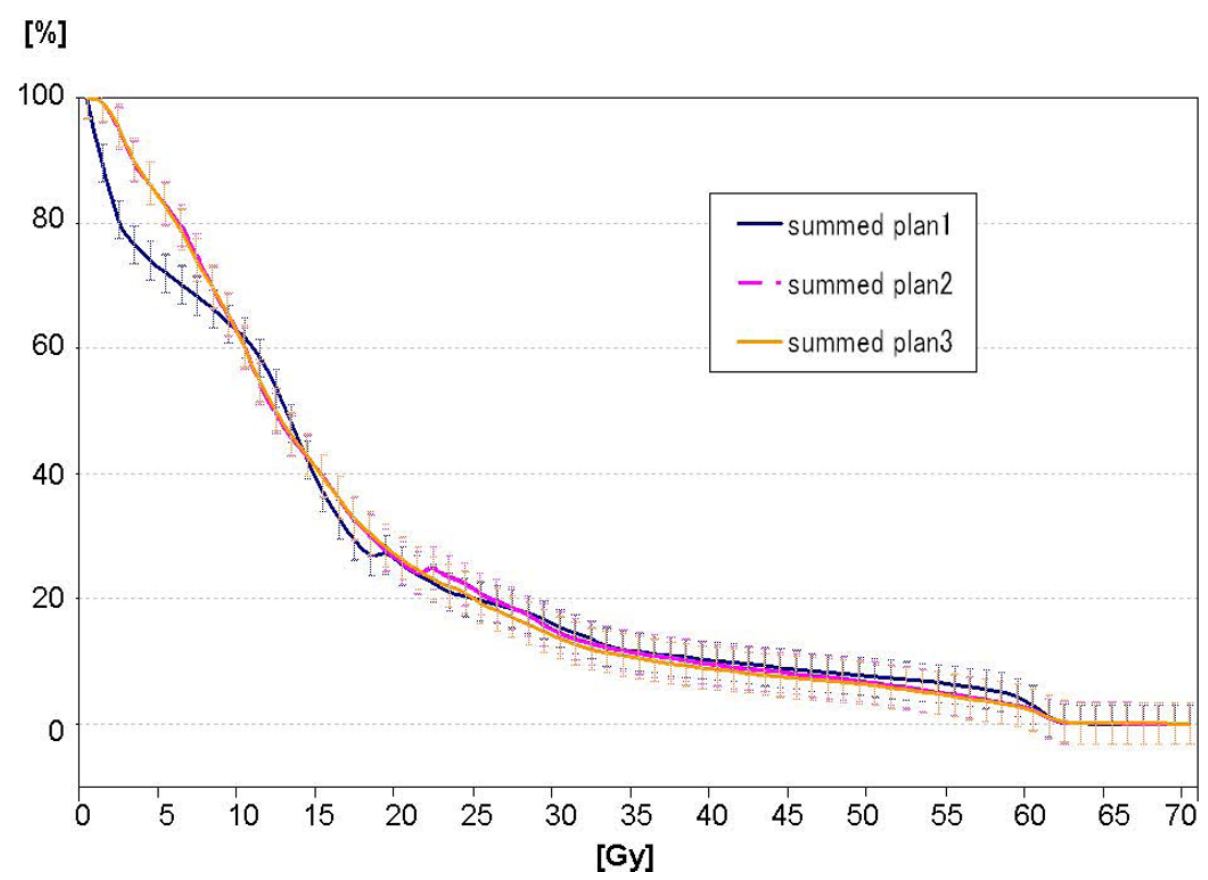

Figure 4 Mean DVH (whole curve) of small bowel PRV in each summed plan.

differences in $\mathrm{D}_{\max }$ or NTCP of small bowel PRV (summed plan 1 vs. summed plan 2 vs. summed plan 3: $\mathrm{D}_{\text {max }}, 41.5 \pm 23.9$ Gy vs. $42.5 \pm 23.4$ Gy vs. $43.8 \pm 24.2$ Gy; NTCP, $4.45 \pm 8.84 \%$ vs. $2.95 \pm 5.87 \%$ vs. $3.30 \pm$ $6.57 \%)$. In 8 patients with presacral or anastomostic recurrence, $\mathrm{D}_{\max }$ of small bowel PRV of summed plan 3 was significantly higher than that of summed plan $2(\mathrm{p}$ $=0.006$ ) but was not significantly higher than that of summed plan 1 (n.s.) (summed plan 1 vs. summed plan 2 vs. summed plan 3: $61.8 \pm 0.6$ Gy vs. $61.1 \pm 1.1$ Gy vs. $64.2 \pm 3.0 \mathrm{~Gy}$ ); however, IMRT could significantly decrease NTCP of small bowel PRV. There was no significant difference in NTCP of small bowel PRV between summed plan 2 and summed plan 3 (summed plan 1 vs. summed plan 2 vs. summed plan 3: $5.42 \pm$ $4.05 \%$ vs. $4.19 \pm 3.56 \%$ vs. $4.49 \pm 3.82 \%, p<0.005$, respectively). The mean DVH of small bowel PRV of summed plan 3 in patients with lateral pelvic lymph node metastasis or perineum recurrence and that in patients with presacral or anastomostic recurrence are shown in Figure 6.

In the present study, although $\mathrm{D}_{\max }$ of small bowel PRV of summed plan 3 was slightly higher than that of summed plan 1 or summed plan $2, \mathrm{~V}_{50}$ of small bowel PRV could be reduced by IMRT, and $V_{30}, V_{40}, V_{60}$, $\mathrm{D}_{\text {mean }}$ and NTCP were not increased even using focal dose escalation.

There was also no significant difference in C.I. between IMRT without dose-painting and IMRT with dose-painting (IMRT without dose-painting boost plan vs. IMRT with dose-painting boost plan: $1.33 \pm 0.10$ vs. $1.29 \pm 0.61(\mathrm{p}=0.115))$.

\section{Discussion}

To our knowledge, there are few reports on PET-guided IMRT for lower gastrointestinal cancer. The reasons why we used this planning method for patients with local recurrent rectal cancer were 1) FDG-PET enabled a recurrent tumor to be distinguished from postoperative scar, 2) FDG-PET could reveal the region with higher malignancy activity and 3) it was not necessary to consider large inter- and intra-fractional motions because of adhesion due to the operation.

There have been several reports on $\mathrm{PET} / \mathrm{CT}$ radiotherapy planning in lung cancer and in head and neck carcinoma. This planning method has been reported to be useful for radiotherapy to delineate target volume. With regard to FDG, there is evidence that FDG-avid regions of a tumor show increased radioresistance in vitro $[18,19]$ and hypoxia in vivo [20]. Therefore, FDG$\mathrm{PET} / \mathrm{CT}$ is also useful for radiotherapy planning to detect the region with high residual potency in GTV to be given priority for treatment with a high dose.

It is difficult to clearly show threshold accumulation between malignancy and non-malignancy by FDG-PET because there is usually inflammation around a malignant tumor, there is penumbra of high accumulation and the normal gut tube has slightly high uptake of 


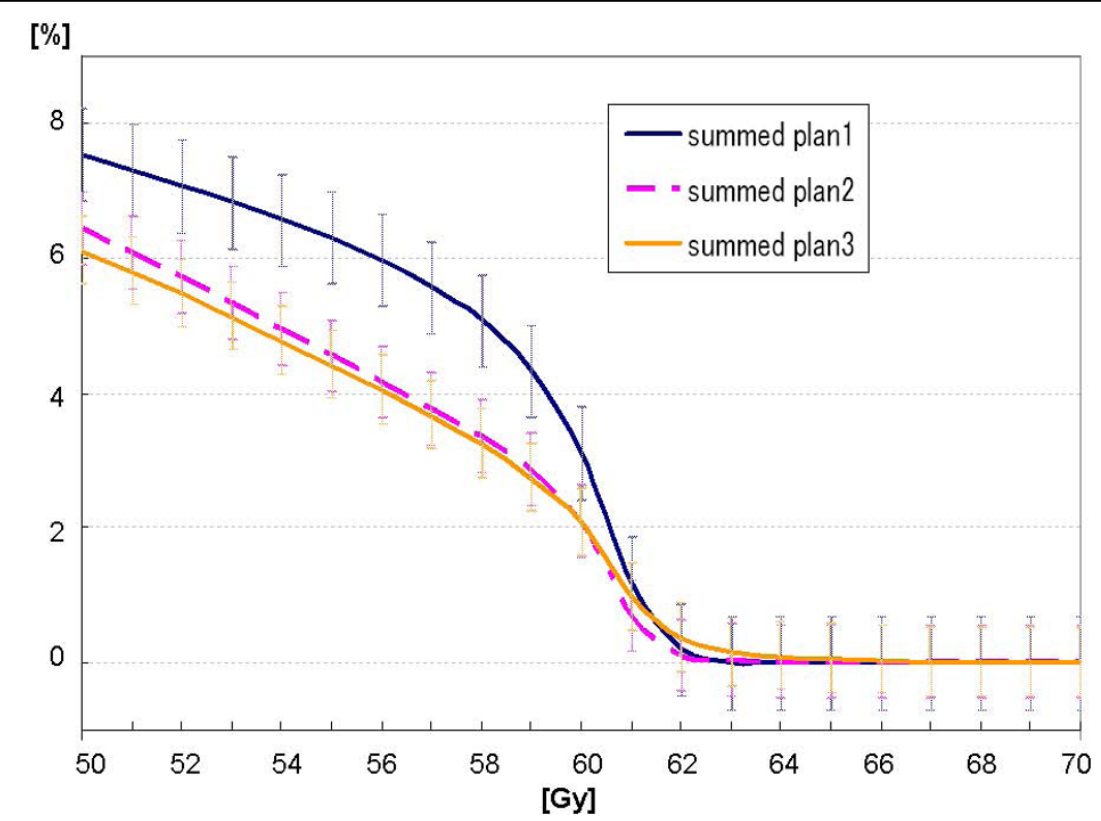

Figure 5 Mean DVH (focused on high dose area (50 to $70 \mathrm{~Gy})$ ) of small bowel PRV in each summed plan.

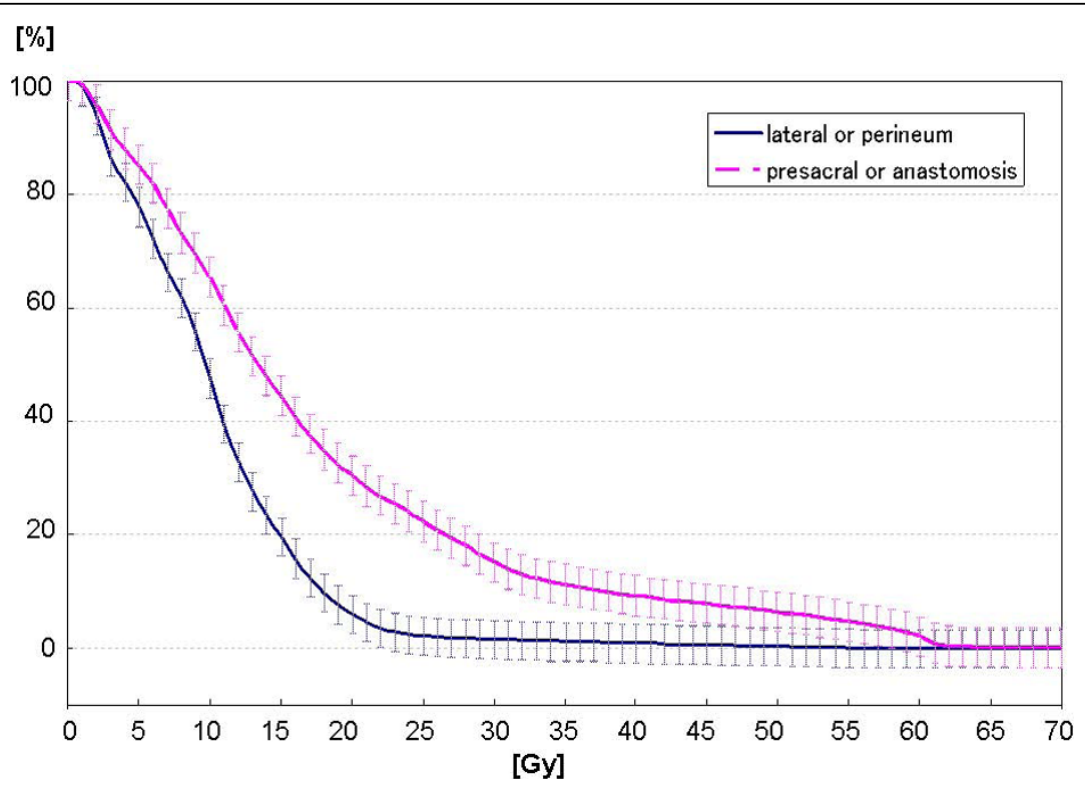

Figure 6 Mean DVH of small bowel PRV of summed plan 3 in patients with lateral pelvic lymph node metastasis or perineum recurrence and that in patients with presacral or anastomostic recurrence. In patients with lateral pelvic lymph node metastasis or perineum recurrence, the mean DVH of small bowel PRV of summed plan 3 was significantly lower than that in patients with presacral or anastomostic recurrence.

FDG. There have been many reports on contouring target volume according to $40 \sim 50 \%$ of maximal SUV value, source-background ratio, and arbitrary SUV value in some malignant tumors [21-25]; however, it remains inconclusive. Bayne et al. pointed out that SUV value had problems with accuracy and reproducibility [26]. In the present study, although we used an arbitrary SUV of
2.0, we consider that it is not a clear border between malignancy and non-malignancy but a region with relatively high malignant potency and with resistance to radiation at $40 \mathrm{~Gy}$ including a subclinical margin like CTV margin. Although SUV of 2.5 3.0 was used as a threshold value between malignancy and non-malignancy in many previous studies, we used SUV of 2.0 as 
the threshold value for BTV based on the fact that patients in the present study had already been irradiated with 40 Gy and based on the fact that Haberkorn et al. reported the mean SUV of recurrent rectal cancer after radiotherapy with 40 Gy to be 1.8 [27].

Furthermore, in the present study, since normal tissues around the GTV were also irradiated with $40 \mathrm{~Gy}$, the possibility that radiation-induced inflammation masked a residual malignant tumor must also be considered. For other tumors such as head and neck cancer and lymphoma $[28,29]$, chemoradiation-induced inflammatory response causes sufficient numbers of false-positive results limiting PET being performed less than 2 months after chemoradiation. It may be inappropriate to use FDG-PET for radiation planning during radiation therapy. Recently, chemotherapy consisting of 5-FU or Capecitabine with or without the addition of Oxaliplatinum has commonly been performed for recurrent rectal cancer. Also, in the present study, all patients underwent concomitant and/or previous chemotherapy with radiation therapy. Findlay et al. mentioned the so-called flare phenomenon that occurs at 1 2 weeks after the initiation of chemotherapy and that can be observed as a marked increase in FDG metabolism in lesions that show response later [30]. We may also have to investigate the appropriate thresholds of FDG accumulation for BTV for each type of chemotherapy. However, in rectal cancer, many investigators revealed that the positive predictive value of FDG-PET assessment of therapy response during or soon after chemoradiation was very high and was not significantly limited by post-chemoradiation changes [31]. The timing of FDG-PET after chemoradiation for the most accurate assessment of tumor response in rectal cancer is controversial. Further larger prospective surveys of the time courses of tumor FDG uptake during and after chemoradiation in rectal cancer are required.

There are other major problems regarding the use of PET/CT for radiation therapy planning: misalignment of the fusion of PET and CT images due to body movement, bowel peristalsis and difference in volume of urine between the transmission scan and emission scan as well as artifacts due to FDG in urine, so-called "hot urine". These problems can be resolved to a large extent by overnight fasting before PET/CT and by starting the emission scan from the position of the pelvis. Moreover, in the present study, a $5-\mathrm{mm}$ circular margin was attached to each target volume and OAR; however, it might not be sufficient to cover such misalignment. It is necessary to investigate such misalignment using on-line imaging (e.g., cone-beam $\mathrm{CT}$ ) before clinical application.

In the present study, since $\mathrm{V}_{30}, \mathrm{~V}_{40}, \mathrm{~V}_{60}, \mathrm{D}_{\text {mean }}$ and NTCP of small bowel PRV were not increased and $V_{50}$ of small bowel PRV could be reduced due to the differences between GTV2 and BTV, focal dose escalation by 6 Gy to regions with SUV above 2.0 using IMRT with dose-painting boost for postoperative local recurrent rectal cancer is considered to be safe. FDGPET-guided IMRT has the possibility of improving local control of postoperative local recurrent rectal cancer without increasing the risk of radiation injury of small bowel PRV. However, although NTCP which reflects account all the DVH data was not increased, $D_{\max }$ of small bowel PRV in the summed plan using focal dose escalation was significantly higher than that in other summed plans. While the differences in mean $D_{\max }$ of small bowel PRV between summed plan 3 and the other plans were only about 2.5 Gy in the present study, $D_{\max }$ of small bowel PRV in summed plan 3 was more than 65.0 Gy in 4 of the 8 patients with anastomotic or presacral recurrence, and NTCP in summed plan 3 in 2 of the 4 patients was more than $10 \%$. Since it is known that the small bowel is a "serial organ" and that the dose at which probability of obstruction or perforation is $50 \%$ within 5 years after treatment (TD50/5) of the small bowel is 55 Gy [32], although NTCP shows that focal dose escalation is acceptable, dose escalation by only 6 Gy from 60 Gy even using PET-guided IMRT is relatively risky. Therefore, if the region of high FDG accumulation is near the OARs, it might be necessary to reduce the degree of dose escalation and/or reduce the volume to increase irradiation dose (e.g., lesion with SUV > 2.5). Alternatively, using IMRT from the beginning of radiotherapy, using a belly board, and inserting a spacer between the recurrent tumor and OARs may further facilitate dose escalation without increasing the risk of radiation injury. When PTV-PET overlaps PRV, we may have to further modify the irradiation dose setting of the overlapping part.

Rectal cancer is known to have many hypoxic fractions [11]. Some studies have provided evidence that hypoxia has a negative impact on tumor response to radiation and other methods of therapy [33-36]. Although we used FDG for radiotherapy planning in this study to determine the region with high tumor cell density, it may be more important for improving the effect of radiotherapy for rectal cancer to determine the hypoxic regions. There are some tracers for detecting a hypoxic region (e.g., $\left[{ }^{18} \mathrm{~F}\right]$ Fluoromisonidazole-3-fluoro-1-(2'-nitro-1'-imidazolyl)-2-propanol ([18 F]FMISO), Cu-diacetyl-bis(N4-methylthiosemicarbazone (Cu-ATSM) and 1-(2-fluoro-1-[hydroxymethyl] ethoxy)methyl-2-nitroimidazole $\left.\left(\left[{ }^{18} \mathrm{~F}\right] \mathrm{FRP} 170\right)\right)$ [37-39]. Although Lin et al. have already reported the effectiveness in head and neck cancer [40], increasing the irradiation dose with IMRT to the hypoxic region may also be effective for treating postoperative recurrent rectal cancer. 


\section{Conclusions}

Our findings suggest that FDG-PET/CT-guided IMRT can facilitate focal dose escalation to regions with SUV above 2.0 while providing normal tissue protection in patients with postoperative local recurrent rectal cancer. However, we do not recommend routine clinical use of focal dose escalation using FDG-PET/CT-guided IMRT. In cases in which the region of high FDG accumulation is near the OARs, careful radiotherapy planning is necessary. Based on the results of this planning study, we will start a clinical phase I/II study of focal dose escalation using PET-guided IMRT for patients with postoperative local recurrent rectal cancer in our institution.

Additional file 1: Supplementary tables.

\section{Acknowledgements}

This study was partially supported by funding from the Japan Radiological Society.

\section{Author details}

'Department of Radiation Oncology, Tohoku University School of Medicine, Sendai, Japan. ${ }^{2}$ Department of Diagnostic Radiology, Tohoku University School of Medicine, Sendai, Japan. ${ }^{3}$ Department of Radiation Oncology, Yamagata University School of Medicine, Yamagata, Japan. ${ }^{4}$ Department of Radiooncology, Heidelberg University, Heidelberg, Germany. ${ }^{5}$ Department of Accelerator Physics and Engineering, National Institute of Radiological Sciences, Chiba, Japan.

\section{Authors' contributions}

$\mathrm{KJ}, \mathrm{ST}, \mathrm{YO}, \mathrm{KN}, \mathrm{HA}$ and SY participated in the design of the study. $\mathrm{KJ}$ and $\mathrm{YT}$ performed the statistical analysis. KJ, TK, TM and LK conceived of the study and participated in its design and coordination. $\mathrm{RU}$ and $\mathrm{YO}$ helped to draft the manuscript. HA, KT, KN, KF and MK acquired data. MM and NM verified and calculated DVH and NTCP. All authors read and approved the final manuscript.

\section{Competing interests}

The authors declare that they have no competing interests.

Received: 4 December 2009 Accepted: 7 April 2010

Published: 7 April 2010

\section{References}

1. Mori T, Takahashi K, Yasuno M: Radical resection with autonomic nerve preservation and lymph node dissection techniques in lower rectal cancer surgery and its results: the impact of lateral lymph node dissection. Langenbecks Arch Surg 1998, 383:409-415.

2. Moriya $Y$, Sugihara $K$, Akasu T, et al: Importance of extended lymphadenectomy with lateral node dissection for advanced lower rectal cancer. World J Surg 1997, 21:728-732.

3. Hida J, Yasutomi M, Fujimoto K, et al: Does lateral lymph node dissection improve survival in rectal carcinoma? Examination of node metastases by the clearing method. J Am Coll Surg 1997, 184:475-480.

4. Bergamaschi $R$, Pessaux $P$, Burtin $P$, et al: Abdominoperineal resection for locally recurrent rectal cancer. Tech Coloproctol 2001, 5:97-102.

5. Weiser MR, Landmann RG, Wong WD, et al: Surgical Salvage of Recurrent Rectal Cancer after Transanal Excision. Dis Colon Rectum 2005, 48:1169-1175.

6. Gunderson LL, Sosin $\mathrm{H}$ : Area of failure found at reoperation following 'curative surgery' for adenocarcinoma of the rectum. Cancer 1974, 34:1278-1292.
7. Tepper JE, O'Connell M, Hollis D, et al: Analysis of surgical salvage after failure of primary therapy in rectal cancer: Results from intergroup study 0114. J Clin Oncol 2003, 21:3623-3628.

8. Kusters M, Beets GL, Velde van de CJ, et al: A comparison between the treatment of low rectal cancer in Japan and the Netherlands, focusing on the patterns of local recurrence. Ann Surg 2009, 249:229-235.

9. Baxter NN, Rothenberger DA, Morris AM, et al: Adjuvant radiation for rectal cancer: do we measure up to the standard of care? An epidemiologic analysis of trends over 25 years in the United States. Dis Colon Rectum 2005, 48:9-15.

10. Pacini $P$, Cionini $L$, Pirtoli $L$, et al: Symptomatic recurrences of carcinoma of the rectum and sigmoid. The influence of radiotherapy on the quality of life. Dis Colon Rectum 1986, 29:865-868.

11. Wendling P, Manz $R$, Thews $G$, et al: Heterogeneous oxygenation of rectal carcinomas in humans: a critical parameter for preperative irradiation? Adv Exp Med Boil 1984, 180:293-300.

12. Eble MJ, Wulf J, van Kampen M, et al: Locally restricted dose escalation in radiotherapy of primary advanced and recurrent rectal cancers. Strahlenther Onkol 1995, 171:77-86.

13. Wong CS, Cummings BJ, Brierley JD, et al: Treatment of locally recurrent rectal carcinoma-results and prognostic factors. Int J Radiat Oncol Biol Phys 1998, 40:427-435.

14. Huebner RH, Park KC, Shepherd JE, et al: A metaanalysis of the literature for whole-body FDG-PET detection of recurrent colorectal cancer. J NuCl Med 2000, 41:1177-1189.

15. Kutcher GJ, Burman $C$, Brewster $L$, et al: Histogram reduction method for calculating complication probabilities for threedimensional treatment planning evaluations. Int J Radiat Oncol Biol Phys 1991, 21:137-146.

16. Lyman JT: Complication probability as assessed from dose-volume histograms. Radiat Res Supp/ 1985, 8:S13-19.

17. Burman C, Kutcher GJ, Emami B, et al: Fitting of normal tissue tolerance data to an analytic function. Int J Radiat Oncol Biol Phys 1991, 21:123-135.

18. Clavo AC, Brown RS, Wahl RL: Fluorodeoxyglucose uptake in human cancer cell lines is increased by hypoxia. J Nucl Med 1995, 36:1625-1632.

19. Burgman $P$, Odonoghue JA, Humm JL, et al: Hypoxia-Induced increase in FDG uptake in MCF7 cells. J Nucl Med 2001, 42:170-175.

20. Pugachev A, Ruan S, Carlin S, et al: Dependence of FDG uptake on tumor microenvironment. Int J Radiat Oncol Biol Phys 2005, 62:545-553.

21. Erdi YE, Rosenzweig K, Erdi AK, et al: Radiotherapy treatment planning for patients with non-small cell lung cancer using positron emission tomography (PET). Radiother Oncol 2002, 62:51-60.

22. Mah K, Caldwell CB, Ung YC, et al: The impact of (18)FDG-PET on target and critical organs in CT-based treatment planning of patients with poorly defined non-small-cell lung carcinoma: a prospective study. Int $J$ Radiat Oncol Biol Phys 2002, 52:339-350.

23. Paulino AC, Koshy M, Howell $R$, et al: Comparison of CT- and FDGPETdefined gross tumor volume in intensity-modulated radiotherapy for head-and-neck cancer. Int J Radiat Oncol Biol Phys 2005, 61:1385-1392.

24. Daisne JF, Duprez T, Weynand B, et al: Tumor volume in pharyngolaryngeal squamous cell carcinoma: comparison at $\mathrm{CT}, \mathrm{MR}$ imaging and FDG PET and validation with surgical specimen. Radiology 2004, 233:93-100.

25. Wang D, Schultz CJ, Jursinic PA, et al: Initial experience of FDG-PET/CT guided IMRT of head-and-neck carcinoma. Int J Radiat Oncol Biol Phys 2006, 65:143-151.

26. Bayne M, MacManus M, Hicks $R$, et al: Can a mathematical formula help define a radiation target volume using positron emission tomography? In regard to Black et al. (Int J Radiat Oncol Biol Phys 60: 1272-1282). Int J Radiat Oncol Biol Phys 2005, 62:299-300.

27. Haberkorn U, Strauss LG, Dimitrakopoulou A, et al: PET studies of fluorodeoxyglucose metabolism in patients with recurrent colorectal tumors receiving radiotherapy. J NuCl Med 1991, 32:1485-1490.

28. Quon A, Fischbein NJ, McDougall IR, et al: Clinical role of 18F-FDG PET/CT in the management of squamous cell carcinoma of the head and neck and thyroid carcinoma. J NuCl Med 2007, 48(Suppl 1):58S-675.

29. Kasamon $Y L$, Jones RJ, Wahl RL: Integrating PET and PET/CT into the riskadapted therapy of lymphoma. J NuCl Med 2007, 48(Suppl 1):19S-27S.

30. Findlay $M$, Young $H$, Cunningham $D$, et al: Noninvasive monitoring of tumor metabolism using fluorodeoxyglucose and positron emission tomography in colorectal cancer liver metastases: correlation with tumor response to fluorouracil. J Clin Oncol 1996, 14:700-708. 
31. de Geus-Oei LF, Vriens D, van Laarhoven HW, et al: Monitoring and predicting response to therapy with 18F-FDG PET in colorectal cancer: a systematic review. J Nucl Med 2009, 50(Suppl 1):43S-54S

32. Emami B, Lyman J, Brown A, et al: Tolerance of normal tissue to therapeutic irradiation. Int I Radiat Oncol Biol Phys 1991, 21:109-122.

33. Höckel M, Knoop C, Schlenger K, et al: Intratumoral pO2 histography as predictive assay in advanced cancer of the uterine cervix. Adv Exp Med Biol 1994, 345:445-450.

34. Höckel M, Schlenger K, Aral B, et al: Association between tumor hypoxia and malignant progression in advanced cancer of the uterine cervix. Cancer Res 1996, 56:4509-4515.

35. Brizel DM, Sibley GS, Prosnitz $L R$, et al: Tumor hypoxia adversely affects the prognosis of carcinoma of the head and neck. Int I Radiat Oncol Biol Phys 1997, 38:285-289.

36. Nordsmark M, Overgaard M, Overgaard J: Pretreatment oxygenation predicts radiation response in advanced squamous cell carcinoma of the head and neck. Radiother Oncol 1996, 41:31-39.

37. Koh WJ, Rasey JS, Evans ML, et al: Imaging of hypoxia in human tumors with [F-18]fluoromisonidazole. Int I Radiat Oncol Biol Phys 1992, 22:199-212.

38. Lewis JS, Sharp TL, Laforest $R$, et al: Tumor uptake of copper-diacetyl-bis( $N$ (4)-methylthiosemicarbazone): effect of changes in tissue oxygenation. J Nucl Med 2001, 42:655-661.

39. Kaneta T, Takai $Y$, Iwata $R$, et al: Initial evaluation of dynamic human imaging using 18F-FRP170 as a new PET tracer for imaging hypoxia. Ann Nucl Med 2007, 21:101-107.

40. Lin Z, Mechalakos J, Nehmeh S, et al: The influence of changes in tumor hypoxia on dose-painting treatment plans based on 18F-FMISO positron emission tomography. Int J Radiat Oncol Biol Phys 2008, 70:1219-1228.

\section{Pre-publication history}

The pre-publication history for this paper can be accessed here: http://www. biomedcentral.com/1471-2407/10/127/prepub

doi:10.1186/1471-2407-10-127

Cite this article as: Jingu et al:: Focal dose escalation using FDG-PETguided intensity-modulated radiation therapy boost for postoperative local recurrent rectal cancer: a planning study with comparison of DVH and NTCP. BMC Cancer 2010 10:127.

\section{Submit your next manuscript to BioMed Central and take full advantage of:}

- Convenient online submission

- Thorough peer review

- No space constraints or color figure charges

- Immediate publication on acceptance

- Inclusion in PubMed, CAS, Scopus and Google Scholar

- Research which is freely available for redistribution 